\title{
Biological and computational evaluation of an oxadiazole derivative (MD77) as a new lead for direct STAT3 inhibitors $\dagger$
}

\author{
Daniela Masciocchi, ${ }^{a}$ Stefania Villa, ${ }^{a}$ Fiorella Meneghetti, ${ }^{a}$ Alessandro Pedretti, ${ }^{a}$ Daniela Barlocco, ${ }^{a}$ \\ Laura Legnani, ${ }^{* b}$ Lucio Toma, ${ }^{b}$ Byoung-Mog Kwon, ${ }^{c}$ Shintaro Nakano, ${ }^{d}$ Akira Asai ${ }^{d}$ and Arianna Gelain ${ }^{* a}$
}

Received 25th January 2012, Accepted 27th February 2012

DOI: $10.1039 / \mathrm{c} 2 \mathrm{md20018j}$

Signal Transducer and Activator of Transcription 3 (STAT3) is a latent cytoplasmic protein overexpressed in various cancer cell lines. STAT3 participates in oncogenesis by stimulating cell proliferation and preventing apoptosis and it has been proven as a suitable target for anticancer therapy. In order to identify direct STAT3 inhibitors, we performed a binding assay on several previously synthesized 1,2,5-oxadiazole derivatives. Among them, compound MD77, $\mathrm{N}$-[4-(4chlorophenyl)-1,2,5-oxadiazol-3-yl]-4-(trifluoromethyl)benzamide, showed a good ability to bind the STAT3-SH2 domain in a dose-dependent manner $\left(\mathrm{IC}_{50}=17.7 \mu \mathrm{M}\right)$. Computational studies were carried out to investigate its binding mode. Moreover, compound MD77 showed a significant antiproliferative activity versus several tumor cell lines. On these bases, compound MD77 was selected as a lead for the future development of direct STAT3 inhibitors.

\section{Introduction}

Signal Transducers and Activators of Transcription (STATs) are a family of cytoplasmic proteins which have two critical roles: to transduce signals through the cytoplasm and to act as transcription factors in the nucleus. Seven STAT family members, encoded by distinct genes, were identified, namely STAT1 to STAT4, STAT5a, STAT5b and STAT6. ${ }^{1}$ They are constituted by several structurally and functionally conserved domains: the $N$-terminal coiled-coil (involved in STAT dimer-dimer interactions), the DNA binding domain (responsible for complex formation between STAT proteins and DNA), the Src homology 2 (SH2) (a linker region required for the recruitment of STAT monomers through reciprocal $p \mathrm{Tyr}-\mathrm{SH} 2$ domain interaction), and finally the $C$-terminal transactivation domain (as

"Dipartimento di Scienze Farmaceutiche "P. Pratesi", Università degli Studi di Milano, Via L. Mangiagalli 25, 20133 Milano, Italy. E-mail: arianna.gelain@unimi.it; Fax: +39-02-503-19359; Tel: +39-02-503-19369 ${ }^{b}$ Dipartimento di Chimica, Università degli Studi di Pavia, Via Taramelli 12, 27100 Pavia, Italy. E-mail: laura.legnani@unipv.it; Fax: +39-038298-7323; Tel: +39-0382-98-7311

${ }^{c}$ Laboratory of Chemical Biology and Genomics, Korea Research Institute of Bioscience \& Biotechnology and Department of Biomolecular Science, Korea University of Science and Technology, Eoun-Dong, Yuseong-gu, Daejeon 305-333, South Korea

${ }^{d}$ Center for Drug Discovery, Graduate School of Pharmaceutical Sciences, University of Shizuoka, 52-1 Yada, Suruga-ku, Shizuoka, 422-8526, Japan $\dagger$ Electronic supplementary information (ESI) available: Synthetic procedures and NMR spectra of MD77, chemical structure of compound $\mathbf{1}$, conformational studies of compound $\mathbf{1}$, docking pose of conformer $\mathbf{C}$ of $\mathbf{M D 7 7}$, mean graphs and dose-response parameters. CCDC reference number 853297. For ESI and crystallographic data in CIF or other electronic format see DOI: $10.1039 / \mathrm{c} 2 \mathrm{md} 20018 \mathrm{j}$ transcriptional activation domain). ${ }^{2}$ The STATs activation cascade involves the phosphorylation of a specific tyrosine residue, which allows the protein dimerization and translocation to the nucleus where STATs are able to modulate gene transcription through a direct binding to DNA.

In particular, STAT3 is constitutively activated in a wide variety of human solid and blood tumors ${ }^{3,4}$ as a result of a deregulation of cytokine receptors, growth factors and Janus kinases (JAK) activity. ${ }^{5,6}$ The inhibition of STAT3 signaling leads to growth arrest and apoptosis of various cancer cell lines, suppresses cancer cell survival, and induces tumor regression, ${ }^{7-9}$ having no effects in normal cells. ${ }^{10}$ For these reasons, STAT3 can be considered a promising target for anticancer therapy. STAT3 signaling can be inhibited through direct interaction of molecules with the protein or by indirect inhibition of the upstream tyrosine kinases or blockage of other factors involved in the activation. ${ }^{11}$ Since the indirect approach is endowed with a poorly specific mechanism of action that could cause important adverse effects,

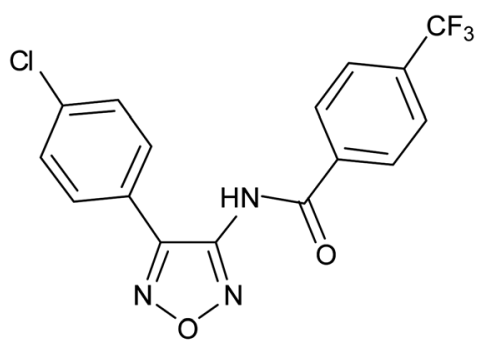

MD77 
the challenge is the discovery of new selective and direct STAT3 inhibitors.

In a previous paper, ${ }^{12}$ we studied, as potential STAT3 inhibitors, three series of 1,2,5-oxadiazoles, bearing at position 3 a ureido, carboxamido, and sulfonamido function, respectively. All the synthesized compounds were evaluated in a dual-luciferase assay at a concentration of $2 \mu \mathrm{M}$, in order to determine their ability to lower STAT3 activity. The interesting results led us to investigate if these compounds were able to directly interact with STAT3. Therefore, they were submitted to the AlphaScreenbased assay, ${ }^{13}$ an in vitro competitive binding test used to identify compounds able to directly inhibit the binding of SH2-containing proteins to their correspondent phosphopeptides, the physiological ligands. Quite unexpectedly, only $N$-[4-(4-chlorophenyl)1,2,5-oxadiazol-3-yl]-4-(trifluoromethyl)benzamide (MD77) proved to be able to significantly interact with the $\mathrm{SH} 2$ domain.

In parallel, MD77 was retested in the dual-luciferase assay ${ }^{14}$ at a higher concentration $(5 \mu \mathrm{M})$ and its anti-proliferative activity was evaluated on a panel of 58 cancer cell lines. Here we report the biological results, the solid state characterization, and the modeling and docking studies of MD77, as a new direct inhibitor of STAT3.

\section{Results and discussion}

\section{Biological studies}

AlphaScreen-based assay. To investigate the direct binding properties of several 3,4-disubstituted-1,2,5-oxadiazoles ${ }^{12}$ to the $\mathrm{SH} 2$ domain we performed the AlphaScreen-based assay, ${ }^{13}$ as described in the Experimental section. In particular, besides STAT3, other SH2-containing proteins, such as STAT1 and Grb2 ("Growth factor receptor-bound protein 2"), having a high degree of sequence homology to STAT3 (78\% and 65\%, respectively) were tested. MD77 was the only compound with a significant activity versus STAT3 $(72.0 \%$ of inhibition at a concentration of $30 \mu \mathrm{M})$. Moreover, it selectively antagonized the STAT3-SH2 domain with respect to the Grb2-SH2 domain (72.0\% versus $10.5 \%$ at $30 \mu \mathrm{M}$ concentration), although it exhibited a higher affinity toward the STAT1-SH2 domain (94.6\% versus $72.0 \%$ at $30 \mu \mathrm{M}$ concentration).

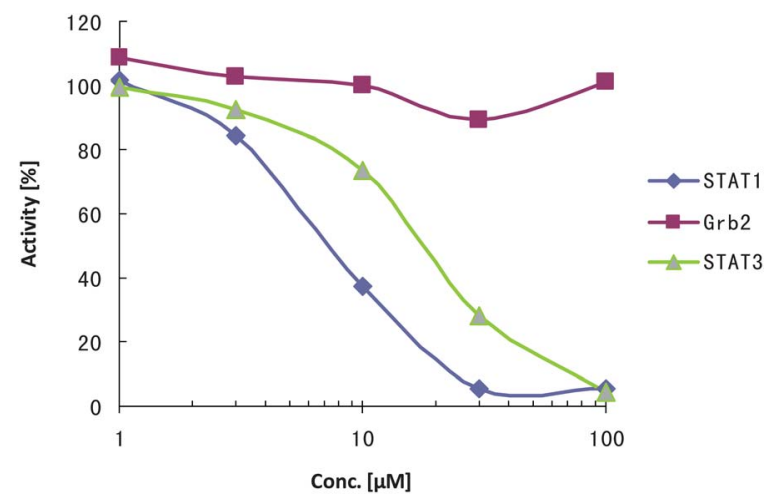

Fig. 1 Dose-response curves of the inhibition of STAT3, STAT1, and Grb2 binding to $p$ Tyr-containing peptides by compound MD77 as determined by AlphaScreen-based assay ( $\%$ of activity versus concentration expressed in logarithmic scale).
Its inhibitory activity proved to be dose-dependent (Fig. 1) with calculated $\mathrm{IC}_{50}$ values of $17.7 \mu \mathrm{M}$ for STAT3, 7.2 $\mu \mathrm{M}$ for STAT1 and higher than $100 \mu \mathrm{M}$ for Grb2.

Dual-luciferase assay. The STAT3 inhibitory activity of MD77 was evaluated in a dual-luciferase assay, ${ }^{14}$ at a higher concentration $(5 \mu \mathrm{M})$ with respect to the published data $(2 \mu \mathrm{M}){ }^{12}$ in human colorectal carcinoma cells HCT-116, characterized by uncontrolled expression of STAT3 (see Experimental section). The results showed that MD77 exhibits an interesting percentage of inhibition $(20 \%)$.

\section{Chemistry}

MD77 was synthesized following slightly modified literature methods. ${ }^{12}$ The synthetic procedure consists of six steps with most of the yields higher than $90 \%$ and only two purifications by flash chromatography (see ESI $\dagger$ ).

\section{X-Ray and conformational analysis of MD77}

The crystallographic structure of MD77 is represented in Fig. 2.

Bond lengths and angles assume the expected standard values. The overall conformation of the compound is defined by four torsional angles: N2-C2-N3-C11 $\left(\tau_{1}\right)$ of $-118(1)^{\circ}, \mathrm{C} 2-\mathrm{N} 3-\mathrm{C} 11-$ $\mathrm{C} 5\left(\tau_{2}\right)$ of $175(1)^{\circ}, \mathrm{N} 3-\mathrm{C} 11-\mathrm{C} 5-\mathrm{C} 6\left(\tau_{3}\right)$ of $-150(1)^{\circ}$ and $\mathrm{C} 2-\mathrm{C} 1-$ $\mathrm{C} 3-\mathrm{C} 13\left(\tau_{4}\right)$ of $22(1)^{\circ}$. The oxadiazole ring is rotated by $25(1)^{\circ}$ and $88(1)^{\circ}$ with respect to the chlorophenyl- and trifluoromethylphenyl moieties, respectively. The latter two rings are perpendicularly oriented with respect to each other, with a dihedral angle of $87(1)^{\circ}$. The analysis of the crystal packing has evidenced the important role played by the halogen atoms in connecting adjacent molecules, through $\mathrm{C} \pi-\mathrm{H} \cdots \mathrm{F}, \mathrm{C} \pi-\mathrm{H} \cdots \mathrm{Cl}$ and $\mathrm{C}=\mathrm{O} \cdots$ $\mathrm{F}$ type contacts, influencing in this way the molecular conformation. Besides, the oxadiazole ring gives rise, in the crystal, to $\pi$ interactions with the chloro-phenyl group of symmetry related molecules along the $b$ axis, indicating the ability of the compound to interact through molecular stacking with a biological counterpart.

The crystallographic structure represents only one of the accessible molecular conformations. Thus, a complete modeling study of MD77 was carried out, considering all the degrees of

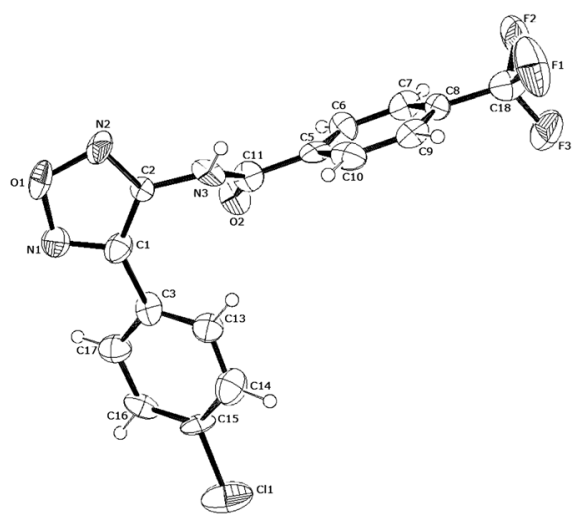

Fig. 2 ORTEP ${ }^{15}$ view of MD77 and the relative arbitrary atomnumbering scheme (thermal ellipsoids at $40 \%$ probability). 


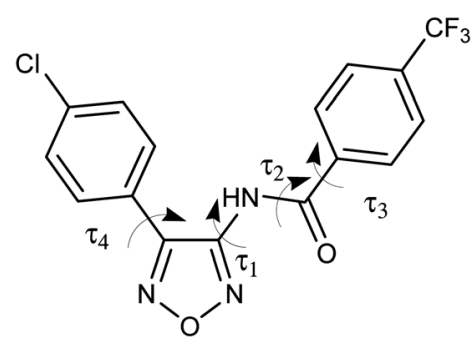

Chart 1 MD77 conformational degrees of freedom.

Table 1 Relative energies, equilibrium percentages, and significant torsional angles of the minimum energy conformations of MD77

\begin{tabular}{llllllrrrr}
\hline & $\begin{array}{l}E_{\text {rel vacuo }} \\
\left(\mathrm{kcal} \mathrm{mol}^{-1}\right)\end{array}$ & $\begin{array}{l}P \text { vacuo } \\
(\%)\end{array}$ & $\begin{array}{l}E_{\text {rel }} \text { water/ } \\
\mathrm{kcal} \mathrm{mol}\end{array}$ & $\begin{array}{l}P \text { water } \\
(\%)\end{array}$ & $\tau_{1}\left({ }^{\circ}\right)$ & $\tau_{2}\left({ }^{\circ}\right)$ & $\tau_{3}\left({ }^{\circ}\right)$ & $\tau_{4}\left({ }^{\circ}\right)$ \\
\hline A & 2.10 & 1.9 & 0.00 & 40.9 & 22 & -177 & -154 & 43 \\
B & 2.49 & 1.0 & 0.17 & 30.6 & 0 & 177 & -158 & -48 \\
C & 0.00 & 65.0 & 0.28 & 25.5 & -126 & 174 & -159 & 34 \\
D & 0.42 & 32.1 & 1.53 & 3.0 & -110 & 174 & -156 & -45 \\
\hline
\end{tabular}

conformational freedom that correspond to the above defined torsional angles (Chart 1) and, in particular, the arrangement of the amidic group with respect to the oxadiazole ring. The geometry optimizations were performed at the B3LYP/ $6-311+G(d, p) l^{2} e^{16}$ and the energy of the optimized conformations was recalculated using a polarizable continuum solvent model (PCM) ${ }^{17}$ to take into account the effect of water.

Four minimum energy conformations were located and in Table 1 their geometrical descriptors $\left(\tau_{1}-\tau_{4}\right)$ are reported, together with their gas-phase and water-solvated energies and the corresponding percentage contributions to the overall population. It is worth pointing out that a mirror image conformation exists for each described conformation; thus, the number of conformations accessible to the molecule is double with respect to those reported.

Conformations A and $\mathbf{B}$ are very similar (Fig. 3), as evidenced by their values of $\tau_{1}, \tau_{2}$, and $\tau_{3}$, the only difference being the orientation of the $p$-chlorophenyl ring $\left(\tau_{4}\right)$. The same occurs for the couple $\mathbf{C}$ and $\mathbf{D}$. The most stable conformation in water (A)
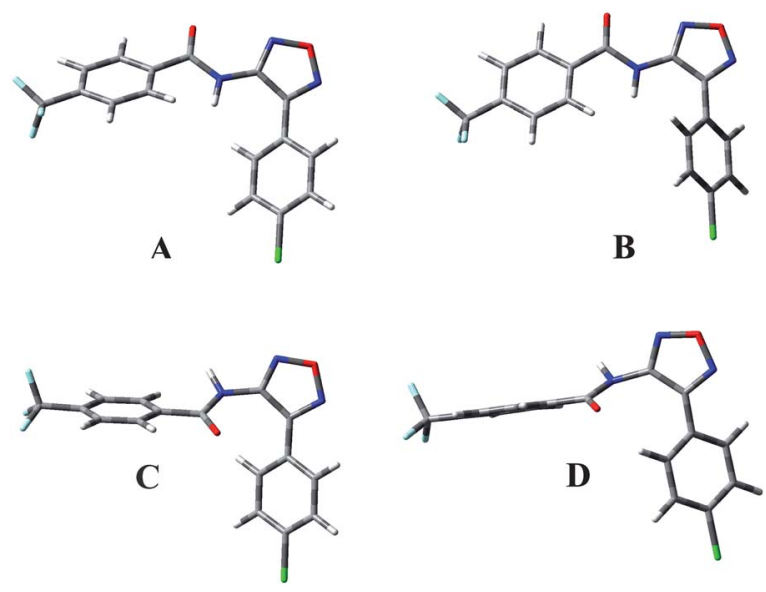

Fig. 3 3D plots of conformations A-D of compound MD77.

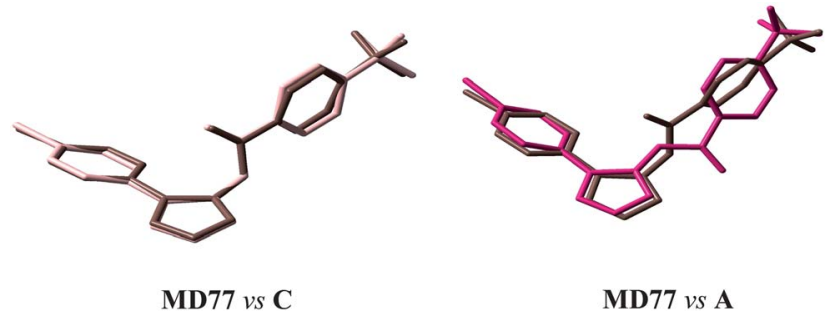

Fig. 4 Superimposition of the crystal structure of compound MD77 (brown) onto the $\mathbf{C}$ (pink) and $\mathbf{A}$ (magenta) conformers obtained through rms fitting of the heavy atoms. Hydrogen atoms are omitted for the sake of clarity.

accounts for $40.9 \%$ of the overall population and, together with conformation $\mathbf{B}$, represents $71.5 \%$, the remaining not negligible $28.5 \%$ being due to the in vacuo preferred conformations $\mathbf{C}$ and $\mathbf{D}$.

A comparison of conformations A-D with the above described crystal structure of MD77 shows that it corresponds to $\mathbf{C}$, which is the most stable conformation in vacuo. In fact, the two structures are characterized by about the same values of $\tau_{1}-\tau_{4}$ and the overlay of their heavy atoms produces an almost perfect superimposition (Fig. 4) with a rms difference value of $0.191 \AA$. By contrast, the comparison of the solid state structure with conformer A gives a rms difference value of $1.067 \AA$. The crystal structure and conformer $\mathbf{A}$ orient their amide function in very different directions (Fig. 4), as evidenced by the significantly different values of $\tau_{1}:-118(1)^{\circ}$ in the crystal versus $22^{\circ}$ in $\mathbf{A}$. The amide function usually represents a key moiety for anchoring a ligand at the binding site of the protein and the ability of MD77 to vary the orientation of the amide under different conditions is worthy of note. The orientation in the crystal is determined by packing interactions, as the molecules form chains through the hydrogen bond $\mathrm{N} 3-\mathrm{H} 3 \cdots \mathrm{O} 2^{\mathrm{I}}$ ( ${ }^{\mathrm{I}}$ at $x, y-1, z$ ), forcing in some way the molecular bending and favoring the conformation having the carbonyl oxygen pointing in the opposite side with respect to the chlorophenyl group.

\section{Docking studies}

Docking studies were performed on MD77 in order to investigate its interaction with the $\mathrm{SH} 2$ domain. In particular, considering the STAT3-MD77 complex and the conformational profile

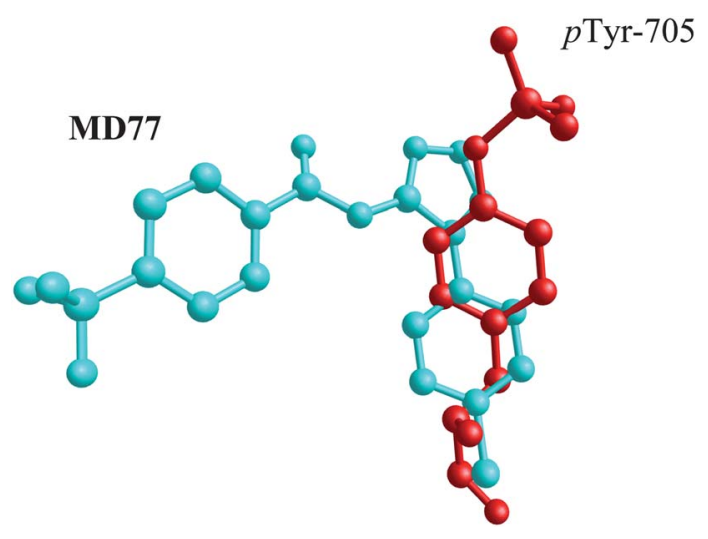

Fig. 5 Comparison of MD77 (cyan) in the docking pose with the $p$ Tyr-705 (red) of the second subunit in the STAT3 dimer. 
of the ligand, the best scored pose showed that the ligand assumes the most stable conformation A. The binding mode of MD77 is comparable to that of phosphorylated Tyr-705, because it involves the same pocket in which $p$ Tyr-705 is inserted when two subunits are assembled in the dimer. The phenyloxadiazole moiety of MD77, colored in cyan, occupies the same pocket (not shown) of $p$ Tyr-705, colored in red (Fig. 5).

This pocket is placed on the protein surface and is surrounded by hydrophilic and polar amino acids to better interact with the negatively charged side chain of the phosphorylated tyrosine, mimed by the phenyloxadiazole moiety in the STAT3-MD77 complex.

As shown in Fig. 6, the favored conformation A of MD77 establishes many hydrogen bond interactions within the binding pocket. In detail, they involve the trifluoromethyl group of the aromatic ring and the guanidine moiety of Arg-595 by three hydrogen bonds, the oxygen of the amidic group and the amine of side chain of Lys-591 by one hydrogen bond, the oxygen and the nitrogen atoms of the oxadiazole ring and the guanidine group of Arg- 609 by two hydrogen bonds and finally the chlorine atom of the aromatic ring with one amidic hydrogen of the Gln635 side chain.

Despite the presence of three aromatic rings in MD77, $\pi-\pi$ interactions were not found in its complex with the macromolecule, due to the absence of aromatic amino acids in the binding pocket. Analyzing the other MD77 complexes obtained by docking, it was possible to identify a pose in which the ligand assumes a conformation referable to the couple $\mathbf{C}-\mathbf{D}$, but its interaction energy is worse than all calculated complexes and the binding mode is much different if compared to the most stable conformation shown above. In particular, the $p$-trifluoromethylphenyl group is partially inserted in the $p \operatorname{Tyr}-705$ pocket while, in the best complex, this pocket is occupied by the oxadiazole ring. In this complex, the oxadiazole ring does not mime the phosphate and stabilizes the complex through

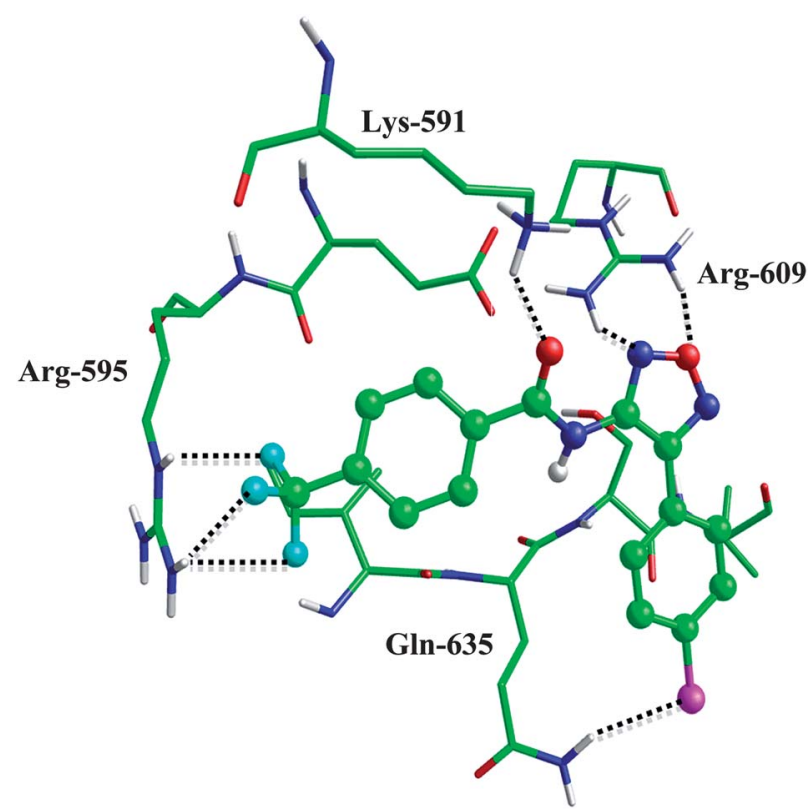

Fig. 6 Main interactions between MD77 and STAT3. The hydrogen bonds are shown as dotted lines.

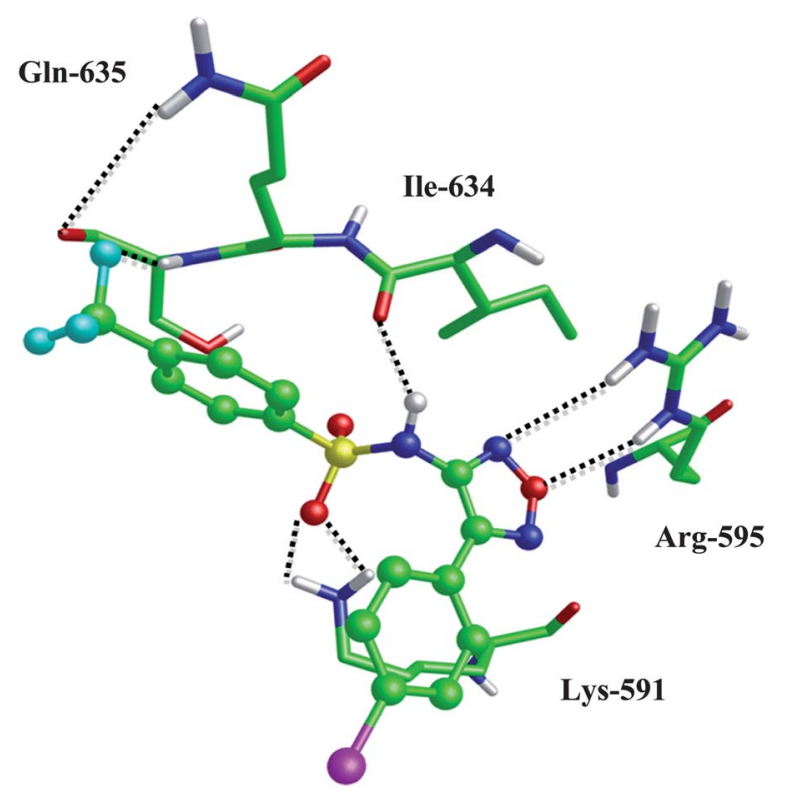

Fig. 7 Main interactions between compound 1 and STAT3. The hydrogen bonds are shown as dotted lines.

hydrogen bonds with Gln-635 and Lys-626 (see ESI $\dagger$ ). Finally, one of the poses of MD77 corresponds to the mirror image of conformation A. It presents a different binding mode in which the $p$ Tyr-705 pocket is not occupied, losing the interaction with Arg-609, but keeping the weak hydrogen bonds between Arg-595 and the trifluoromethyl group.

It should be noted that the sulfonamido bioisoster (1) of MD77, which was found inactive both in the AlphaScreen and in the dual-luciferase assays, has a different conformational behavior with respect to our lead compound. In fact, none of the localized conformations of $\mathbf{1}$ shows a relative arrangement of the aromatic groups similar to that displayed in the four geometries of MD77 (see ESI $\dagger$ ). Thus, the identification of its binding mode through docking calculations seemed to be important to clarify the essential features for an efficient interaction with STAT3 (Fig. 7).

Considering the STAT3-compound 1 complex, the pose and interacting conformation of the ligand are much different from those shown by MD77. In particular, compound $\mathbf{1}$ is unable to occupy the $p$ Tyr-705 pocket and the oxadiazole ring interacts with Arg-595 instead of Arg-609, which is the key residue involved in the MD77 complex and in the formation of the salt bridge with the phosphate group when the protein dimerizes. Despite the different binding mode, the compound $\mathbf{1}$ complex is stabilized by an extended network of hydrogen bonds, shown in Fig. 7. In the docked conformation of compound 1 the two benzene rings are perpendicular, the angle between them $\left(89^{\circ}\right)$ being significantly higher than the corresponding value $\left(28^{\circ}\right)$ found for MD77 in the best score pose.

\section{Anti-proliferative assay}

In order to verify the anti-proliferative activity of compound MD77, cell proliferation assays were performed by $\mathrm{NCI}$ (Bethesda, USA) under the Developmental Therapeutic Program (DTP) to determine its effect on tumor cells growth. MD77 was 

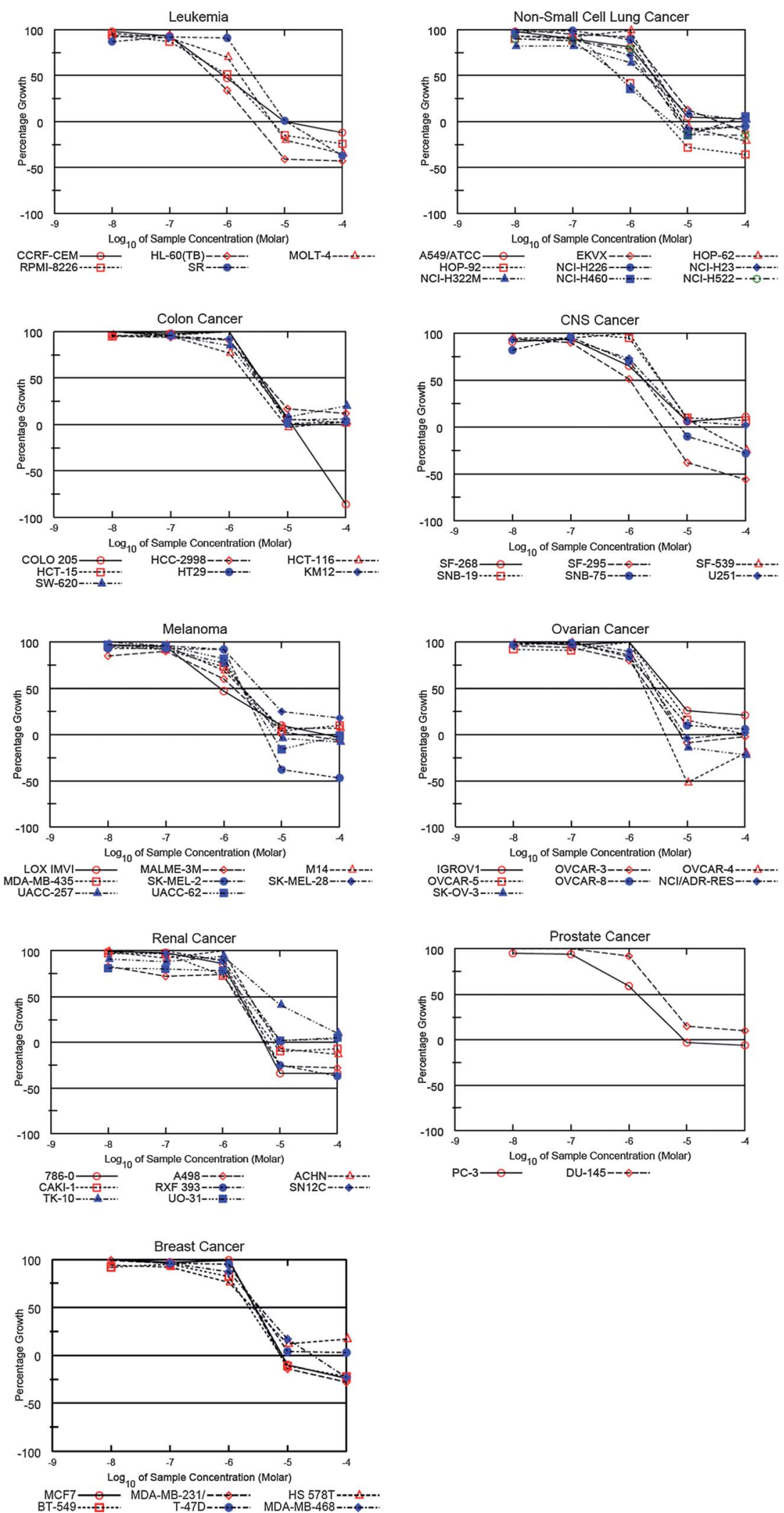

Fig. 8 Dose-response curves showing the percentage growth inhibition of compound MD77 in panel/cell lines (data obtained from NCI in vitro disease oriented tumor cell screen). 
exposed to a panel of 58 human tumor cell lines, derived from 9 cancer cell types, and it was firstly tested at a single dose of $10 \mu \mathrm{M}^{18}$ (see Experimental section).

Since it exhibited a significant growth inhibition value, it was subjected to a complete analysis at five different doses for 48 hours. The data are expressed as dose-response parameters $\mathrm{GI}_{50}$, TGI and $\mathrm{LD}_{50}$ referred to MD77 molar concentration that produces $50 \%$ of growth inhibition, total growth inhibition and $50 \%$ of cytotoxicity, respectively (see ESI $\dagger$ ). In these assays MD77 exhibited a good profile of inhibitory activity on cell proliferation, with $\mathrm{GI}_{50}$ values ranging from $6.75 \times 10^{-6} \mathrm{M}$ (renal cancer, TK10) to $5.46 \times 10^{-7} \mathrm{M}$ (leukemia, HL60TB), and showing TGI values lower than $1.00 \times 10^{-4} \mathrm{M}$ in most of the cell lines (the dose-response parameters expressed as mean graph are reported in the ESI $\dagger$ ).

The dose-response curves showing the activity of MD77 on the panel/cell lines are represented in Fig. 8. As expected, MD77 inhibits the growth of the cell lines which are known to overexpress STAT3 (for instance HCT116, DU145, and MDA-MB231), although several cell lines, such as HL60TB (leukemia cells) and HOP-92 (non-small cells lung cancer), are the most sensitive.

\section{Conclusions and future perspectives}

The ability to target the STAT3-SH2 domain of a series of oxadiazole derivatives was evaluated in order to identify new, direct inhibitors. One compound (MD77) proved to be able to significantly interact with the SH2 domain: in the AlphaScreen binding assay, it showed an interesting dose-response profile with an $\mathrm{IC}_{50}$ value of $17.7 \mu \mathrm{M}$. This result highlighted its potential as a protein-protein interaction inhibitor. In addition, MD77 exhibited a significant activity $(20 \%$ inhibition at $5 \mu \mathrm{M})$ in a dual-luciferase assay. To investigate the conformational behavior and the binding mode of MD77, modeling and docking studies were performed. The latter evidenced that in the best scored pose of the STAT3-MD77 complex, MD77 assumes the most stable conformation calculated in water. It should be noted that the binding mode of MD77 is comparable to that of $p$ Tyr705 , when the latter is involved in the formation of the STAT3 dimer. The computational data were supported by crystallographic studies. Finally, MD77 displayed a significant growth inhibitory activity on a number of tumor cell lines. In the light of these interesting results, MD77 emerged as a lead for the development of a new series of derivatives that is actually underway.

\section{Experimental}

\section{AlphaScreen-based assay}

AlphaScreen is a bead-based nonradioactive assay system for detecting biomolecular interactions in a microtiter plate format. Binding of biological partners brings donor and acceptor beads into close proximity and as a result, a fluorescent signal between 520 and $620 \mathrm{~nm}$ is produced. The AlphaScreen-based assays ${ }^{13}$ were performed in a final reaction volume of $25 \mu \mathrm{L}$ of the assay buffer containing $10 \mathrm{mM}$ HEPES-NaOH (pH 7.4), $50 \mathrm{mM}$ $\mathrm{NaCl}, 1 \mathrm{mM}$ EDTA (pH 8.0), 0.1\% NP-40, and $10 \mathrm{ng} \mu \mathrm{L}^{-1} \mathrm{BSA}$ in a 96-well microtiter plate at $25{ }^{\circ} \mathrm{C}$. Phospho-Tyr ( $p$ Tyr) peptide probes used in this study were 5-carboxyfluorescein (FITC)-GpYLPQTV for STAT3, FITC-GpYDKPHVL for
STAT1, and FITC-PSpYVNVQN for Grb2. Firstly, $75 \mathrm{nM}$ of each $\mathrm{SH} 2$-containing protein was incubated with the test compound for $15 \mathrm{~min}$. Each protein sample was then incubated for 90 min with $50 \mathrm{nM}$ of its corresponding FITC- $p$ Tyr peptide, and mixed with streptavidin coated donor beads and anti-FITC acceptor beads simultaneously before detection at $570 \mathrm{~nm}$ using EnVison Xcite (PerkinElmer).

\section{Dual-luciferase assay}

Cell culture. The cancer cell lines were obtained from American Type Culture Collection. Human breast cancer cell lines (MDA-MB-468 and MDA-MB-231) and the human colon cancer cell line (SW620) were maintained in RPMI 1640 (Gibco/ BRL). Another human colon cancer cell line (HCT-116) was maintained in McCoy's 5A (Gibco/BRL). All culture media were supplemented with $10 \%$ heat-inactivated fetal bovine serum (Gibco/BRL). Cell cultures were maintained at $37{ }^{\circ} \mathrm{C}$ under a humidified atmosphere of $5 \% \mathrm{CO}_{2}$ in an incubator.

Transient transfection and dual-luciferase assays. ${ }^{14}$ HCT-116 cells were seeded at a density of $10 \times 10^{5}$ cells in $100 \mathrm{~mm}^{2}$ culture plate. The cells were co-transfected with pSTAT3-TA-Luc $(27 \mu \mathrm{g}$ per plate) and an internal control plasmid pRL-TK $(9 \mu \mathrm{g}$ per plate) containing the Renilla luciferase gene. All plasmids used in this experiment were purchased from Promega. The transfection was carried out using TransFectin (Bio-Rad), according to the manufacturer's protocol. After $5 \mathrm{~h}$ of transfection, the cells were trypsinized and seeded onto sterilized black bottom 96-well plates at a density of $1 \times 10^{4}$ cells per well. On the following day, cells were treated with test compounds and incubated for $24 \mathrm{~h}$. Firefly and Renilla luciferase activities were measured using a dual-light reporter gene assay kit (Promega) on Wallac Victor2 (Perkin-Elmer, Inc., Wellesley, MA). Renilla luciferase activity was determined to calibrate transfection efficiency and cytotoxicity of chemicals. Relative STAT3 activity was calculated by dividing the firefly luciferase activity with Renilla luciferase activity in each transfection experiment. The values of STAT3 inhibitory activity were the means of 3 experiments and the maximum deviation from the mean was less than $10 \%$.

Cell proliferation assay. Cells were seeded at a density of 5000 cells per well in 96-well plates in RPMI 1640 or McCoy's medium containing 10\% FBS. They were replenished with fresh complete medium containing either test compound or $0.1 \%$ DMSO. After incubation for 24 or $48 \mathrm{~h}$, the cell proliferation reagent WST-1 (Roche Applied Science) was added to each well. WST-1 formazan was quantitatively measured at $450 \mathrm{~nm}$ using an enzymelinked immunosorbent assay reader (Bio-Rad).

\section{Crystallography}

Crystals of MD77 were obtained from an ethanol solution at room temperature as white platelets. The intensity data were collected on an Enraf Nonius CAD-4 diffractometer with Mo K $\alpha$ radiation $(\lambda=0.71073 \AA)$ at room temperature. The lattice parameters were determined by least-squares refinements of 25 high angle reflections. Crystal system: monoclinic $(P c)$, cell dimensions (̊̊): $a=11.115(3), b=5.008(3), c=14.112(5), \beta=$ 
92.28(1). Final $R$ indices $[I>2 s(I)] R 1=0.044, \mathrm{w} R_{2}=0.057$ for 1549 independent reflections. The structure was solved by direct methods ${ }^{19}$ and the refinement was carried out with SHELX-97.20 All non-H-atoms were refined anisotropically. The amidic $\mathrm{H}$ position was detected in a difference Fourier synthesis and refined with isotropic thermal factors, while the other hydrogen atoms were introduced at calculated positions in their described geometries and allowed to ride on the attached carbon atom with fixed isotropic thermal parameters (1.2 Ueq. of the parent carbon atom) $\dagger$

\section{Conformational analysis}

The calculations were carried out using the Gaussian09 program package. $^{21}$ The conformational space of compound MD77 was explored through optimizations at the B3LYP level with the $6-311+\mathrm{G}(\mathrm{d}, \mathrm{p})$ basis set. ${ }^{16}$ Compound 1 was modeled at the same level as above (B3LYP/6-311+G(2df,p) level for the $S$ atom). All the degrees of conformational freedom were considered paying particular attention to the possible arrangements of the amidic group with respect to the oxadiazole ring. The energy of the optimized conformations was recalculated in water using a polarizable continuum model (PCM). ${ }^{17}$

\section{Molecular docking}

The calculations were performed to evaluate the binding affinity of MD77 and compound 1 with the STAT3-SH2 domain. STAT3 structure, co-crystallized with a DNA fragment, was downloaded from the Protein Data Bank ${ }^{22}$ (PDB ID 1BG1 ${ }^{23}$ ) and was dimerized applying the transformation matrix as reported in the PDB file. The model was completed adding the hydrogens in two steps: (1) to STAT3, applying the algorithm for proteins and (2) to DNA, applying the algorithm for nucleic acids. In both cases, we used the features included in the VEGA $\mathrm{ZZ}$ package. ${ }^{24}$ Atom charges (the Gasteiger-Marsili method ${ }^{25}$ ) and potentials (CHARMM 22 for proteins ${ }^{26}$ and nucleic acids ${ }^{27}$ ) were assigned to the obtained structure. Finally, the model was optimized through a conjugate gradients minimization (30000 steps) in order to reduce the high-energy sterical interactions. In order to preserve the experimental data, atom constraints were applied to the protein and the DNA backbones. This step was carried out by NAMD $2.8^{28}$ integrated in the VEGA ZZ graphic environment. Before running GriDock, the grid maps required to evaluate the docking score were calculated, selecting the atoms included in a sphere of $12 \AA$ radius centered on phosphorylated Tyr-705 (PTR-705 in the PDB file), which is known to play a pivotal role in the STAT3 dimerization and activation. This phase was carried out by AutoGrid 4 interfaced to VEGA ZZ. MD77 and compound 1 were docked by GriDock/AutoDock ${ }^{29}$ using the genetic algorithm search and generating 20 possible solutions. All these complexes were minimized by NAMD (conjugate gradients, 10000 steps), keeping the atoms fixed outside from the spheroid defined by a layer of $12 \AA$ thickness around the ligand.

\section{Cell proliferation assays}

Compound MD77 was sent to the National Cancer Institute (NCI) in Bethesda, Maryland (USA) and screened in a panel of 58 human tumor cell lines, derived from nine neoplastic cancer types (leukemia, lung, colon, CNS, melanoma, ovarian, renal, prostate, and breast cancers) to test its anti-proliferative activity. The screening was a two-stage process, beginning with the evaluation of the compounds against 58 human tumor cell lines at a single dose of $10 \mu \mathrm{M}$. These preliminary results are expressed as percentages of growth inhibition of treated cell lines per panel when compared to untreated control cells. MD77 exhibited a significant growth inhibition and thus it was evaluated against the 58 cell panel at five concentration levels.

Methodology of the anti-proliferative assay. The anti-proliferative assay was performed according to the US NCI protocol. ${ }^{18}$ Briefly, the human tumor cancer cell lines of the screening panel were grown in RPM 1640 medium containing 5\% fetal bovine serum and $2 \mathrm{mM}$ L-glutamine. Cells were inoculated into 96-well microtiter plates in $100 \mu \mathrm{L}$ of complete medium at densities ranging from 5000 to 40000 cells per well. The microtiter plates containing the cells were incubated for $24 \mathrm{~h}$ at $37{ }^{\circ} \mathrm{C}, 5 \% \mathrm{CO}_{2}$, $95 \%$ air and $100 \%$ relative humidity prior to addition of the experimental drug. MD77 was solubilized in dimethyl sulfoxide at 400 -fold the desired final maximum test concentration and stored frozen prior to use. At the time of drug addition, an aliquot of frozen concentrate was thawed and diluted twice to the desired final maximum test concentration with a complete medium containing $50 \mu \mathrm{g} \mathrm{mL}^{-1}$ gentamicin. Additional four-, 10fold serial dilutions were made to provide a total of five drug concentrations plus control. Aliquots of $100 \mu \mathrm{L}$ of these different drug dilutions were added to the appropriate microtiter wells already containing $100 \mu \mathrm{L}$ of medium, resulting in the required final drug concentrations.

Following the addition of the compound, the plates were incubated for an additional $48 \mathrm{~h}$ at $37{ }^{\circ} \mathrm{C}, 5 \% \mathrm{CO}_{2}, 95 \%$ air, and $100 \%$ relative humidity. Cells were fixed by the gentle addition of $50 \mu \mathrm{L}$ of cold $50 \%$ (w/v) trichloroacetic acid (TCA) and incubated for 60 minutes at $4{ }^{\circ} \mathrm{C}$. After washing with tap water and air drying, Sulforhodamine B (SRB) solution $(100 \mu \mathrm{L})$ at $0.4 \%$ $(\mathrm{w} / \mathrm{v})$ in $1 \%$ acetic acid was added to each well, and plates were incubated for 10 minutes at room temperature. After removing the unbound dye by washing with $1 \%$ acetic acid, the bound stain was subsequently solubilized with $10 \mathrm{mM}$ Trizma base and the absorbance was measured on a microplate reader.

Dose-response parameters $\left(\mathrm{GI}_{50}, \mathrm{TGI}, \mathrm{LD}_{50}\right)$ were calculated as reported in the NCI protocol. ${ }^{18}$

\section{Acknowledgements}

The authors thank Dr Kenji Matsuno for his fruitful support, CILEA (Milan) for the allocation of computer time, the Developmental Therapeutics Program, Division of Cancer Treatment and Diagnosis, National Cancer Institute ${ }^{18}$ (Bethesda, USA) for conducting the cell proliferation assays and the Universities of Milan and Pavia for the financial support (PUR 2008 and FAR grants, respectively). B.-M. K. was supported by the National Research Foundation of Korea (NRF-2011-0015768).

\section{Notes and references}

1 J. E. Darnell, Science, 1997, 277, 1630-1635.

2 J. Bromberg and J. E. Darnell, Oncogene, 2000, 19, 2468-2473. 
3 L. B. Mora, R. Buettner, J. Seigne, J. Diaz, N. Ahmad, R. Garcia, T. Bowman, R. Falcone, R. Faircloug, A. Cantor, C. Muro-Cacho, S. Livingstone, J. Karras, J. Pow-Sang and R. Jove, Cancer Res., 2002, 62, 6659-6666.

4 G. Niu, T. Bowman, M. Huang, S. Shivers, D. Reintgen, A. Daud, A. Chang, A. Kraker, R. Jove and H. Yu, Oncogene, 2002, 21, 7001-7010.

5 L. Song, J. Turkson, J. G. Karras, R. Jove and E. B. Haura, Oncogene, 2003, 22, 4150-4165.

6 U. M. Wegenka, J. Buschmann, C. Lutticken, P. C. Heinrich and F. Horn, Mol. Cell. Biol., 1993, 13, 276-288.

7 J. Turkson and K. Siddiquee, Cell Res., 2008, 18, 254-267.

8 S. O. Rahaman, P. C. Harbor, O. Chemova, G. H. Barnett, M. A. Vogelbaum and S. J. Haque, Oncogene, 2002, 21, 8404-8413.

9 J. R. Grandis, S. D. Drenning, Q. Zeng, S. C. Watkins, M. F. Melhem, S. Endo, D. E. Johnson, L. Huang, Y. He and J. D. Kim, Proc. Natl. Acad. Sci. U. S. A., 2000, 97, 4227-4232.

10 W. M. Burke, X. Jin, H. J. Lin, M. Huang, R. Liu, R. Reynolds and J. Lin, Oncogene, 2001, 20, 7925-7934.

11 D. Masciocchi, A. Gelain, S. Villa, F. Meneghetti and D. Barlocco, Future Med. Chem., 2011, 3, 367-397.

12 D. S. Shin, D. Masciocchi, A. Gelain, S. Villa, D. Barlocco, F. Meneghetti, A. Pedretti, Y.-M. Han, D. C. Han, M. Y. Han, B.-M. Kwon, L. Legnani and L. Toma, Med. Chem. Commun., 2010, 1, 156-164.

13 Y. Uehara, M. Mochizuki, K. Matsuno, T. Haino and A. Asai, Biochem. Biophys. Res. Commun., 2009, 380, 627-631.

14 B. A. Sherf, S. L. Navarro, R. R. Hannah and K. V. Wood, Promega Notes, 1996, 57, 2-8.

15 C. K. Johnson, ORTEP 11, Report ORNL-5138, OakRidge National Laboratory, TN, 1976.

16 (a) C. Lee, W. Yang and R. G. Parr, Phys. Rev. B: Condens. Matter Mater. Phys., 1988, 37, 785-789; (b) A. D. Becke, J. Chem. Phys., $1993,98,5648-5652$.

17 (a) E. Cancés, B. Mennucci and J. Tomasi, J. Chem. Phys., 1997, 107, 3032-3042; (b) M. Cossi, V. Barone, R. Cammi and J. Tomasi, Chem. Phys. Lett., 1996, 255, 327-335; (c) V. Barone, M. Cossi and J. Tomasi, J. Comput. Chem., 1998, 19, 404.

18 National Cancer Institute, http://dtp.nci.nih.gov.
19 A. Altomare, M. C. Burla, M. Camalli, G. Cascarano, C. Giacovazzo, A. Gagliardi and G. Polidori, J. Appl. Crystallogr., 1994, 27, 435.

20 G. M. Sheldrick, SHELX-97, University of Göttingen, Germany.

21 M. J. Frisch, G. W. Trucks, H. B. Schlegel, G. E. Scuseria, M. A. Robb, J. R. Cheeseman, G. Scalmani, V. Barone, B. Mennucci, G. A. Petersson, H. Nakatsuji, M. Caricato, X. Li, H. P. Hratchian, A. F. Izmaylov, J. Bloino, G. Zheng, J. L. Sonnenberg, M. Hada, M. Ehara, K. Toyota, R. Fukuda, J. Hasegawa, M. Ishida, T. Nakajima, Y. Honda, O. Kitao, H. Nakai, T. Vreven, J. A. Montgomery, Jr, J. E. Peralta, F. Ogliaro, M. Bearpark, J. J. Heyd, E. Brothers, K. N. Kudin, V. N. Staroverov, R. Kobayashi, J. Normand, K. Raghavachari, A. Rendell, J. C. Burant, S. S. Iyengar, J. Tomasi, M. Cossi, N. Rega, J. M. Millam, M. Klene, J. E. Knox, J. B. Cross, V. Bakken, C. Adamo, J. Jaramillo, R. Gomperts, R. E. Stratmann, O. Yazyev, A. J. Austin, R. Cammi, C. Pomelli, J. W. Ochterski, R. L. Martin, K. Morokuma, V. G. Zakrzewski, G. A. Voth, P. Salvador, J. J. Dannenberg, S. Dapprich, A. D. Daniels, Ö. Farkas, J. B. Foresman, J. V. Ortiz, J. Cioslowski, and D. J. Fox, Gaussian 09, Revision A.02, Gaussian, Inc., Wallingford CT, 2009.

$22 \mathrm{http} / / / \mathrm{www} . \mathrm{pdb}$. org/.

23 S. Becker, B. Groner and C. W. Müller, Nature, 1998, 934, 145-151.

24 A. Pedretti, L. Villa and G. Vistoli, J. Mol. Graphics Modell., 2002, 21, $47-49$.

25 J. Gasteiger and M. Marsili, Croat. Chem. Acta, 1980, 53, 601-614.

26 A. D. MacKerell Jr, D. Bashford, M. Bellott, R. L. Dunbrack Jr, J. D. Evanseck, M. J. Field, S. Fischer, J. Gao, H. Guo, S. Ha, D. Joseph-McCarthy, L. Kuchnir, K. Kuczera, F. T. K. Lau, C. Mattos, S. Michnick, T. Ngo, D. T. Nguyen, B. Prodhom, W. E. Reiher, III, B. Roux, M. S. chlenkrich, J. C. Smith, R. Stote, J. Straub, M. Watanabe, J. Wiorkiewicz-Kuczera, D. Yin and M. Karplus, J. Phys. Chem. B, 1998, 102, 3586-3616.

27 N. Foloppe and A. D. MacKerell Jr, J. Comput. Chem., 2000, 21, 86104.

28 J. C. Phillips, R. Braun, W. Wang, J. Gumbart, E. Tajkhorshid, E. Villa, C. Chipot, R. D. Skeel, L. Kale and K. Schulten, J. Comput. Chem., 2005, 26, 1781-1802.

29 D. S. Goodsell and A. J. Olson, Proteins: Struct., Funct., Genet., 1990, 8, 195-202. 